\title{
A SPECTROSCOPIC STUDY OF BINARY STAR PLANETARY
}

\section{NEBULAE}

\author{
J.R. WALSH \\ Space Telescope European Coordinating Facility, European Southern Observatory, \\ Karl-Schwarzschild-Strasse 2, D8046 Garching bei München, Germany \\ N.A. WALTON \\ Department of Physics \& Astronomy, University College London, \\ Gower Street, London WC1E 6BT, England
}

and

\section{S.R. POTTASCH}

Kapteyn Laboratorium, Rijksuniversiteit Groningen, Postbus 800, 9700 AV Groningen, The Netherlands

\begin{abstract}
A comprehensive spectrophotometric survey of the central stars of planetary nebulae with known or suspected binary star nuclei (BSPN) is underway. The aims of this programme are: to determine the nature of the companion to the ionising star of the nebula; to estimate the spectroscopic distance from the spectral type and magnitude of the companion and thence to determine the luminosity of the hot star; to compare the morphologies and excitation/abundance characteristics of BSPN with those of PN produced by single star evolution. The advantage of the distance determined from this simple method is that it is independent of any assumptions about the planetary nebula, in contrast to most other PN distance estimators.

To date 75 percent of known or suspected BSPN have been observed with the $2.5 \mathrm{~m}$ Isaac Newton Telescope and the $1.5 \mathrm{~m}$ ESO spectroscopic telescope at low and intermediate dispersions. A selection of preliminary results from the programme is presented, highlighting the binary central stars of NGC 246 and Abell 65.
\end{abstract}

\section{Preliminary results from the programme}

Preliminary analysis of low $(12 \AA)$ and higher $(1.6 \AA)$ resolution spectra of the hot central star of NGC 246 and it's nearby visual companion, give the companion's visual magnitude, as $\mathrm{m}_{\mathrm{v}}=14.4 \pm 0.1$ mags. The $\mathrm{B}-\mathrm{V}$ colour is 1.09 mag. leading to an estimate of $\mathrm{K} 2 \mathrm{~V}-\mathrm{K} 3 \mathrm{~V}$. The identified stellar features imply a somewhat earlier spectral class of K0 V. Our preliminary classification of the cool star as type K0 V, with an absolute magnitude of $\mathrm{M}_{\mathrm{V}}=5.9$ (Jaschek \& Jaschek, 1990), leads to a distance to NGC 246 of 500 pc.

Our observations of the central star spectrum of Abell 65 show it to be quite remarkable, having an inverted Balmer decrement. It shows weak HeI and HeII lines with a strong C III,N III feature at $4645 \AA$. Recently Acker \& Stenholm (1990) have reported on their observations of the central star of HFG 1 . On the basis of it's spectrum, containing an inverted Balmer decrement with lines of He I, He II, $\mathrm{C}_{\mathrm{III}}$ and $\mathrm{N}$ III in emission, they proposed that the central star of HFG 1 may be a member of the intermediate polar class of magnetic cataclysmic variables. Our spectrum of nucleus of Abell 65 shows it to be similar to that of HFG 1's nucleus, and hence it may also be related to the cataclysmic variables, having a high density, hot accretion disk surrounding the central star system.

\section{References}

Acker, A., Stenholm, B. 1990, A\&A, 233, L21

Jaschek, C., Jaschek, M. 1990, 'The Classification of Stars', Cambridge University Press 